\title{
Impact de L'installation des Camps des Refugiés, Retournés et Déplacés sur L'exploitation des Ressources Ligneuses dans la Région de Diffa
}

\author{
Rabiou Habou, \\ Mahamane Mansour, \\ Issaharou-Matchi Issiaka, \\ Université de Diffa, Niger \\ Mahamane Ali, \\ Université Abdou Moumouni de Niamey, Université de Diffa, Niger
}

Doi:10.19044/esj.2019.v15n36p118 URL:http://dx.doi.org/10.19044/esj.2019.v15n36p118

\section{Résumé}

La région de Diffa située dans la vallée du Bassin du Lac Tchad à l'extrème Est de la République du Niger, fait face à la persécution de la secte Boko Haram. Les populations frontalières avec la République fédérale du Nigéria, principales victimes fuyant la guerre sont contraints de se réfugier vers la région de Diffa. L'installation des camps des réfugiés a occasionné des profondes modifications au niveau du paysage déjà affecté par la désertification. La présente étude dont l'objectif est d'analyser l'impact de l'installation des camps de réfugiés sur les ressources ligneuses et la dynamique paysagère a été conduite au niveau des camps des réfugiés des communes de Gueskérou et Chétimari. Des placettes de $2500 \mathrm{~m}^{2}$ ont été délimitées sur des transects radiaires au niveau des camps de réfugiés et des villages autochtones. Dans chaque placette, les mesures dendrométriques ont été réaliséés sur les espèces ligneuses. Les facteurs de pression tels que la coupe, l'émondage et l'écorçage ont été relévés sur tous les arbres. Des enquêtes sur l'utilisation des ressources ligneuses ont été effectuées. Les données satellitaires (Landsat) ont été collectées afin d'analyser la dynamique paysagère avant et après l'installation des camps. L'analyse des résultats montre que les espèces ligneuses, les plus écologiquement importantes sont Balanites aegyptiaca, Acacia tortilis, Boscia senegalensis, Acacia senegal et Leptadenia pyrotechnica. Toutes les espèces ligneuses sont soit utilisées comme bois de service dans la confection des abris ou comme bois énergie. L'analyse des indicateurs de pression montre que les fréquences de coupe, d'émondage et d'écorçage sont plus importantes dans les premières auréoles des camps de réfugiées. L'analyse de la conversion de l'occupation montre que pour l'ensemble des communes, les sols nus sont en progression et une 
régression des superficies de la steppe arbustive, de la forêt galerie et une extension des steppes arbustives dégradées et la formation de dunes de sable. L'impact de l'installation des camps de réfugiés sur l'environnement constitue une préoccupation dans la région de Diffa, une région déjà affectée par la désertification et le changement climatique.

Mots clés: Camps Des Réfugiés, Ressources Ligneuses, Dynamique Paysagère, Diffa, Niger

\title{
Impact of the Installation of Refugee Camps, Returned and Displaced on the Exploitation of Wood Resources in the Region of Diffa
}

\author{
Rabiou Habou, \\ Mahamane Mansour, \\ Issaharou-Matchi Issiaka, \\ Université de Diffa, Niger \\ Mahamane Ali, \\ Université Abdou Moumouni de Niamey, Université de Diffa, Niger
}

Abstract

The region of Diffa is located in the Lake Chad basin valley in the eastern part of Niger Republic. This region is facing persecution by the terrorist Boko Haram sect and border populations with the Federal Republic of Nigeria are forced to flee to this region because of this conflict. Consequently, the refugee camps settlement has caused profound changes in the region landscape already affected by desertification.Therefore, The aim of the present study is to analyse the impact of the refugee camps settlement on woodland-based ecosystem servicies and landscape dynamics in Gueskérou and Chétimari communes. Plots of $2500 \mathrm{~m}^{2}$ have been delimited on radial transects at refugee camp and indigenous village levels. In each plot, dendrometric measurements were taken on the woody species. Pressure factors such as cutting, pruning and debarking on trees were recorded. Surveys were conducted in order to collect the information of woodland-based ecosystem used servicies. The Landsat data were collected to analyse the landscape dynamics before and after the setting up of the camps. Results analysis shows that the most ecologically important woody species are Balanites aegyptiaca, Acacia tortilis, Boscia senegalensis, Acacia senegal and 
Leptadenia pyrotechnica. The woody species are used as woodland-based ecosystem servicies such as pillars in the shelters setting up and as fuelwood. Analysis of pressure indicators shows that cutting, pruning and debarking frequencies are higher in the first halos of refugee camps. The result of the occupation conversion shows that the bare soils are in full progression and regression is noted on the the shrub steppe, the gallery forest areas while an extension of the shrub steppes degradation and the formation of sand dunes is recorded in the two communes. The environmental impact of the settling refugee camps is a distress in this region already affected by desertification, land degradation and climate change.

Keywords: Refugee Camps, Woodland-Based Ecosystem Servicies, Landscape Dynamics, Diffa, Niger

\section{Introduction}

Depuis 2013, le conflit lié à la secte Boko Haram dans le bassin du lac Tchad a provoqué le déplacement de milliers de réfugiés, des déplacés internes et des retournés contraint d'abandonner le droit à l'accès aux terres et aux actifs agricoles (UNHCR, 2013). Cette situation a provoqué des besoins humanitaires importants dans une région déjà confrontée à la pauvreté et à la dégradation environnementale (UNHCR, 2017 ; Adamou et Adamou, 2018). Cependant, l'essartage pour l'ouverture des camps ou des sites et les prélèvements du bois énergie et ou de service pour les charpentes des abris se sont accentués au détriment des formations végétales environnantes (UICN/UNHCR, 2005). Cet afflux massif des personnes a donné lieu à la création de plusieurs camps et sites de réfugiés ou déplacés internes dans les différentes communes de la région de Diffa (Mamadou, 2016). L'empreint écologique liée à la présence des réfugiés ou des rapatriés sur l'environnement est visible et difficile à effacer (Diallo, 2015). En particulier dans les environs des sites de déplacés des communes de Chétimari et Gueskérou (MHA, 2017), une pression toujours plus importante s'exerce sur les ressources naturelles comme la terre, le bois et le pâturage et les capacités de charge de ces écosystèmes arides sont largement dépassées (MHA, 2017). Le manque de bois de chauffe et de construction se manifeste de plus en plus aux environs de la zone d'accueil des populations déplacées (Richard, 1998). Pourtant ces formations végétales fournissent une variété de bois et de produits forestiers non ligneux tels que les plantes médicinales, les fleurs, les fruits, les feuilles et les racines qui contribuent à l'économie des ménages et renforcent la sécurité alimentaire ainsi que la conservation de la diversité biologique. De nombreuses personnes dans la région de Diffa dépendent directement de ces ressources forestières pour leur subsistance et leur revenu (Mamadou, 2016 ; Diallo, 2015). 
La destruction du couvert végétal par les réfugiés et les déplacés prive le sol de sa protection naturelle avec des conséquences néfastes pour le régime des eaux (baisse de la nappe phréatique) et accélère fortement l'érosion hydrique et éolienne (FAO, 2012 ; MESU/DD, 2014). La perte du matériel organique et de la terre fine dans le sol risque de provoquer des dégâts quasi irréversibles au niveau des écosystèmes (Maazou et al., 2017 ; AFD, 2018). La dégradation des écosystèmes se poursuit par la déforestation (déboisement) et le surpâturage (émondage des arbres) par une forte concentration du bétail (Sinsin, 1993 ; Rabiou et al., 2017). La capacité de régénération est d'autant réduite que l'aridité est prononcée. Si rien n'est fait pour les contenir ou pour proposer des alternatives, ce qui commence par une exploitation bénigne de diverses ressources peut prendre rapidement l'allure d'une dévastation étendue et irréversible (Youn, 1985 ; Mamadou, 2016). Face à cette, il n'est simplement pas envisageable de ne pas intervenir. La présente étude s'inscrit dans une logique de production des données et des indicateurs pouvant servir de base à l'élaboration des plans de gestion durable. Les résultats pourraient permettre une meilleure appréhension de l'état actuel des ressources ligneuses et l'empreint écologique marquée par la présence des personnes déplacées afin d'élaborer des mesures appropriées d'atténuations ou d'éventuelles stratégies de réhabilitation des sites. L'objectif de l'étude est d'analyser l'impact de l'installation des camps de réfugiés sur les ressources ligneuses et la dynamique paysagère.

\section{Materiel Et Méthodes \\ Sites d'étude}

L'étude a été conduite dans deux communes de la région de Diffa au Niger. Il s'agit des communes de Gueskérou et Chétimari. La Commune Rurale de Gueskérou est située au centre du département de Diffa, à l'extrême sud-est du Niger sur la route Nationale $\mathrm{N}^{\circ} 1$, à $1400 \mathrm{~km}$ de Niamey, la capitale et à $40 \mathrm{~km}$ à l'Est de la ville de Diffa (chef-lieu de région) (PDC, 2010) (Figure 1). Elle couvre une superficie de $1971 \mathrm{~km}^{2}$ et compte une population estimée à 46034 habitants (INS, 2012). Elle fait partie des Communes de la région qui accueillent le plus grand nombre de réfugiés et déplacés. En effet, plus de 77961 personnes (soit environ le double de la population locale) ont été installées dans la commune et réparties dans 21 sites, dont la plupart sont situés le long de la Route Nationale 1 (RN1), axe Niamey-N'Djamena (Tchad) traversant la Région (UNHCR, 2017). D'après Saadou (1990), la Commune Rurale de Gueskerou est soumise au climat Nord-Sahélien.

La Commune de Chétimari est située dans la Région de Diffa, Département de Diffa, à l'extrême Sud-Ouest sur la Route Nationale $\mathrm{N}^{\circ} 1$, à $1345 \mathrm{~km}$ de Niamey, la capitale. Elle couvre une superficie de $2566 \mathrm{~km}^{2}$ représentant $37 \%$ de la superficie du département et 1,83\% de la superficie 
totale de la région de Diffa (PDC, 2012). Elle est limitée au nord par les communes de N'Gourti et Kabaléwa, au sud par la République Fédérale du Nigeria (Etat de Borno) sur plus de $20 \mathrm{~km}$, matérialisés par la rivière Komadougou Yobé, à l'est par les communes de Gueskérou et Diffa et à l'ouest par la commune de Maïné Soroa. La commune de Chétimari renferme d'importantes potentialités constituées de trois (3) forêts classées (Gagamari, Malaminari et Kalgounam) totalisant 980 ha et des forêts protégées (MESU/DD, 2014).

Le cumul pluviométrique annuel se situe entre 200 et $400 \mathrm{~mm}$ d'eau. L'Humidité relative varie entre 19 et 70\% RH (Kiari et al., 2017). La végétation de ces deux communes est composée essentiellement de steppes (arbustive, herbeuse et arborée) et des galeries forestières le long des cours d'eau. La principale activité des populations est la culture du riz et du poivron dans la vallée de la rivière Komadougou Yobé qui borde le sud de ces deux Communes, constituant ainsi la frontière naturelle avec le Nigéria (Mamadou, 2016).

Les principales activités sources de revenus des ménages dans la zone d'étude sont l'agriculture, l'élevage, le petit commerce avec la Nigeria voisin. On peut aussi noter que beaucoup des jeunes des villages de ces deux communes frontalières avec le Nigéria ont rejoint le mouvement Boko Haram.

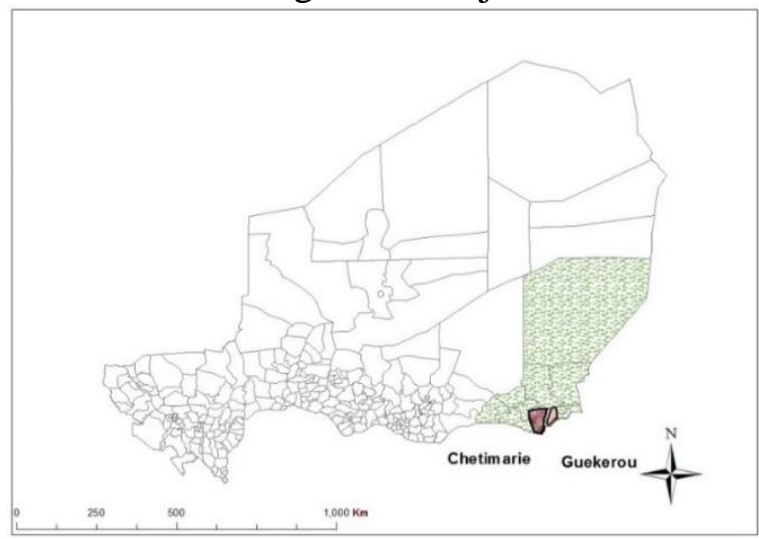

Figure 1. Situation géographique des sites d'étude (La carte doit être beaucoup plus élaborée et illustrée avec tous les détails avec une légende)

\section{Echantillonnage et collecte des données Inventaire forestier}

Dans le cadre de la présente étude, une approche pludisciplinaire a été adoptée. Au niveau de chaque commune, des sites réprésentatifs ont été identifiés. Il s'agit des camps des réfugiés, des déplacées internes, des rétournés et des villages autochtones. Ainsi, au niveau de la Commune de Chétimari, les camps de Boudouri, de Maina Kaderi, de Sayam forage et le village de Gamari ont été prospectés. Au niveau de la Commune de 
Gueskérou, ce sont les camps de Djalori, Kindjandi et le village de Garin Wanzam qui ont fait l'objet de prospections.

Les enquêtes ethnobataniques basées sur des focus groupes ont été conduites au niveau de chaque site. Ces enquêtes ont permis de collecter les informations sur la dynamique de la végétation et les principales ressources ligneuses utilisées pour la construction des abris par les réfugiés et toutes autres formes d'utilisation de ces ressources. Les principales rubriques du questionnaire sont : (i) Alimentation ; (ii) Bois de service ; (iii) Bois énergie ; (iv) Médicinales ; (v) Fourragères.

$\mathrm{Au}$ niveau de chaque site, un inventaire des espèces ligneuses a été effectué afin d'analyser les impacts de prélèvement et déterminer les indicateurs de pression. La méthode des transects radiaires a été utilisée au niveau de chaque site (Figure 2). Sur chaque transect, des placettes de 2500 $\mathrm{m}^{2}$ ont été délimitées avec une équidistance de $200 \mathrm{~m}$. Les premières placettes de chaque transect ont été délimitées à $100 \mathrm{~m}$ du camp ou du village. Au niveau de chaque site, 10 placettes ont été réalisées sur quatre (4) transects, soit 40 placettes par site. Les mesures dendrométriques ont été portées sur tous les arbres adultes (diamètres, hauteur et deux diamètres perpendiculaires du houppier). Les facteurs de pression tels que les traces d'émondage, l'écorçage et la coupe ont été relevés sur tous les arbres.

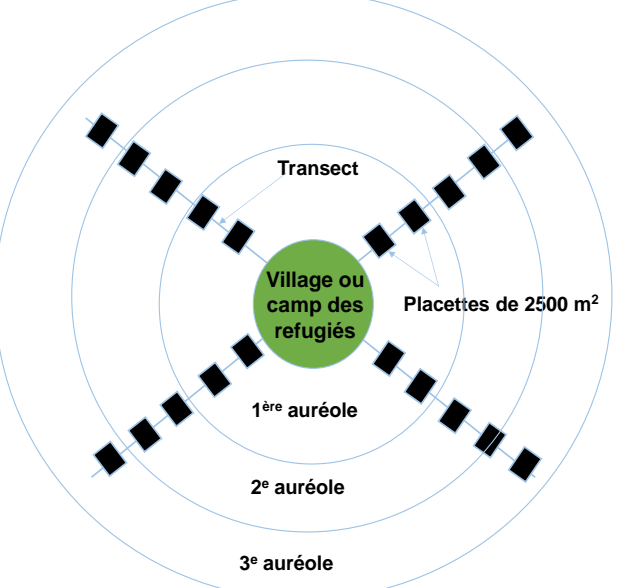

Figure 2. Dispositif de l'inventaire

\section{Données géograpgiques}

Les images satellitaires provenant de capteurs Thematic Mapper (TM5) pour l'année 2002, Enhanced Thematic Mapper plus (ETM+) pour l'année 2013 et landsat 8 OLI pour l'année 2017 ont été collectées. Le mois d'août a été choisi afin de minimiser les confusions entre les classes. Au niveau de chaque commune, 250 points ont été collectés durant le mois d'août 2017 pour soupporter le traitement des données satellitaires et une classification 
dite supervisée. Aussi, $70 \%$ des points ont été utilisés pour la classication et $30 \%$ pour la validation (Mahamane, 2015).

\section{Analyse et traitement des données}

Les données collectées ont été saisies puis traitées à l'aide du tableur Excel. Le répertoires des différentes espèces et de leurs utlisations ont été constitués. Afin d'analyser l'intensité de pression, les données sur les caractéristiques dendrométriques et les facteurs de pression ont été analysées suivant les auréoles. Les premières auréoles sont proches des camps, les secondes auréoles sont intermédiaires et les troisièmes auréoles sont loins des camps. De premières auréoles aux troisièmes, le gradient de préssion diminue. L'indice des valeurs d'importance (combinaison de la densité, de la surface terrière et recouvrement) a été utilisé pour determiner l'imortance écologique de chaque espèce. La surface terrière, la hauteur de Lorey, le recouvrement moyen de chaque site ont été calculés selon les dispositions auréolaires. Les fréquences de coupes, d'écorçages et d'émondages ont été calculées suivant les auréoles. Une analyse en composantes principales $(\mathrm{ACP})$ a été réalisée à l'aide de Logiciel Minitab 14 afin de récapituler les interactions entre les facteurs de pression et les caractéristiques dendrométriques suivant les auréoles.

Pour l'analyse de la dynamique de l'occupation des sols, les operations de pretraitement (correction atmospherique, geometrique et radiometrique) ont été réalisées sur quatre images landsat (Figure 3) avec le Logiciel QGIS et ERDAS imagine. L'amélioration radiométrique consiste à corriger les effets des différents artefacts qui perturbent la mesure radiométrique. La correction géométrique a été effectuée pour rectifier les images satellites de manière à les rendre superposables à d'autres images ou à des documents cartographiques de référence (Samaali, 2011).

Les images utilisées ont été ainsi prealablement georeferencées (Projection UTM, Zone 36S), rectifiées et orthorectifiées par le fournisseur. Les bandes individuelles ont été compilées et les communes délimitées après le mosaiquage par l'outil "Subset and Chip' de Erdas imagine. La légende a été developée sur la base de la nomenclature d'occupation des terres publiée par UNEP/FAO (1994) et les informations des signatures spectrales des images satellites (Suleiman et al., 2014). La classification supervisée a été utilisée dans la présente étude du fait de sa robustesse et de son aspect pratique (Figure 3). Ainsi, l'algorithme selectioné pour la classification est le maximun de vraisemblance. Pendant les travaux de la classification google Earth platforme et RADAR (sentinel1) ont été utilisés pour une meilleure interprétation des données. Dans le but de minimiser l'effet de pixels isolés au niveau des resultats de la classification, le filtrage spatial a été utilisé à 1 aide 
de l'algorithme filtre majoritaire de taille 5 x 5 kernels (Mahamane et al., 2015).

Pour valider les resultats de l'occupation du sol, les 75 points de contrôle collectés ont été utilisés pour tester la fiabilité de la classification. La fiabilité de la classification ou précision globale a été calculée en divisant le nombre des cellules qui ont une classification correcte par le nombre des cellules dans la matrice de confusion (Singh, 1986 ; Enaruvbe et Atedhor, 2015). Aussi, l'indice de Kappa calculé montre que tous les resultats de l'occupation du sol ont des valeurs superieures à Chetimari $89 \%$ en 2002, 93\% en 2017 et Gueskérou $81 \%$ en 2013 et $87 \%$ en 2017) 80\% donc acceptable et fiable (Manandhar et al., 2009 ; Congalton, 1991 ; Forkuo et Frimpong, 2012, Mahamane, 2015).

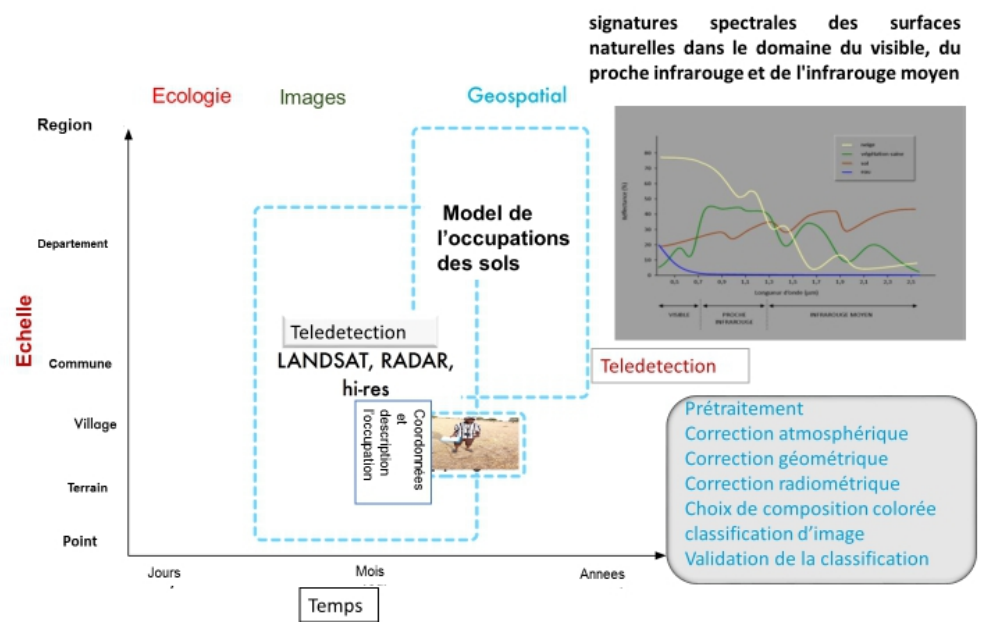

Figure 3. Rectificatif de la méthodologie

\section{Résultats}

\section{Importance écologique des ligneux}

L'analyse de l'indice des valeurs d'importance montre que les espèces les plus écologiquement importantes sont Balanites aegyptiaca, Acacia tortilis, Boscia senegalensis, Acacia senegal et Leptadenia pyrotechnica. Cependant, d'un site à un autre, on constate des petites variations. En effet, l'espèce Acacia tortilis est plus importante au niveau du sire de Boudouri. Les sites de Maina Kaderi et Malam Gamari sont dominés par Balanites agyptiaca. Les espèces Cadaba farinosa et Cordia sinensis ne sont observées qu'au niveau du site de Malam Gamari. Ce dernier s'est également distingué des autres sites par une richesse spécifique plus eleveé (13 espèces ligneuses) contre 11 à Boudouri et 9 espèces à Maina Kaderi (Tableau 1). 
Tableau 1. Importance écologique et compostion floristique des sites

\begin{tabular}{|c|c|c|c|c|}
\hline \multirow{2}{*}{ Espèces } & \multicolumn{4}{|c|}{ IVI } \\
\cline { 2 - 5 } & Camp de & Camp de Maina & Malam & Global \\
\hline Balanites & 106,1 & 132,8 & 106,3 & 115,04 \\
\hline Acacia tortilis & 130,9 & 117,1 & 60,7 & 102,91 \\
\hline Boscia & 3,2 & 0,6 & 80,7 & 28,14 \\
\hline Acacia senegal & 38,7 & 6,8 & 15,5 & 20,35 \\
\hline Leptadenia & 0,6 & 34,9 & 0,8 & 12,08 \\
\hline Ziziphus & 2,3 & 2,1 & 18,8 & 7,7 \\
\hline Maerua crassifolia & 10,1 & 2,5 & 4,7 & 5,77 \\
\hline Commiphora & 4,8 & - & 3,4 & 2,71 \\
\hline Faidherbia albida & 0,6 & 1,1 & 4,5 & 2,07 \\
\hline Calotropis procera & 2,2 & 2,2 & 0,3 & 1,56 \\
\hline Cadaba farinosa & - & - & 3,1 & 1,04 \\
\hline Bauhinia rufescens & 0,6 & - & 1 & 0,53 \\
\hline Cordia sinensis & - & - & 0,3 & 0,11 \\
\hline Total général & 300 & 300 & 300 & 300 \\
\hline
\end{tabular}

\section{Usages des principales espèces ligneuses}

Les enquêtes conduites au niveau des camps des réfugiés et des villages autochtones montrent que toutes les espèces répertoriées, en plus des autres formes d'utilisation, sont utilisées comme bois énergie. Sur les 14 espèces recensées, 10 sont utilisées dans les traitements médicinaux. Les espèces Acacia senegal et Balanites aegyptiaca sont utilisées dans toutes les catégories d'usages. Toutes les espèces hormis Acacia nilotica, Faidherbia albida, Maerua crassifolia et les Ziziphus spp. sont utilisées dans la construction des abris pour les réfugiées (Tableau 2). Les espèces utilisées comme plantes alimentaires sont Acacia senegal, Balanites aegyptiaca, Boscia senegalensis, Cordia sinensis, Prosopis juliflora, Ziziphus mauritiana, Hyphaene thebaica et Ziziphus spina-christi.

Tableau 2. Espèces ligneuses et leurs utilisations

\begin{tabular}{|c|c|c|c|c|c|}
\hline Nom d'espèces & Alimentation & Bois de service & Bois énergie & Médicinales & Fourragères \\
\hline Acacia nilotica & & & $\mathrm{x}$ & $\mathrm{X}$ & \\
\hline Acacia senegal & $\mathrm{x}$ & $\mathrm{x}$ & $\mathrm{x}$ & $\mathrm{X}$ & $\mathrm{x}$ \\
\hline Acacia tortilis & & $\mathrm{x}$ & $\mathrm{x}$ & $\mathrm{X}$ & $\mathrm{x}$ \\
\hline Balanites & $\mathrm{x}$ & $\mathrm{x}$ & $\mathrm{x}$ & $\mathrm{X}$ & $\mathrm{x}$ \\
\hline Boscia & $\mathrm{x}$ & $\mathrm{x}$ & $\mathrm{x}$ & $\mathrm{X}$ & \\
\hline Commiphora & & $\mathrm{x}$ & $\mathrm{x}$ & & $\mathrm{x}$ \\
\hline Cordia sinensis & $\mathrm{x}$ & $\mathrm{x}$ & $\mathrm{x}$ & $\mathrm{X}$ & \\
\hline Faidherbia albida & & & $\mathrm{x}$ & $\mathrm{X}$ & $\mathrm{x}$ \\
\hline Maerua crassifolia & & & $\mathrm{x}$ & & $\mathrm{x}$ \\
\hline Leptadenia & & $\mathrm{x}$ & $\mathrm{x}$ & $\mathrm{X}$ & \\
\hline
\end{tabular}




\begin{tabular}{|c|c|c|c|c|c|}
\hline Prosopis juliflora & $\mathrm{x}$ & $\mathrm{x}$ & $\mathrm{x}$ & & \\
\hline Ziziphus & $\mathrm{x}$ & & $\mathrm{x}$ & $\mathrm{X}$ & $\mathrm{x}$ \\
\hline Hyphaene thebaica & $\mathrm{x}$ & $\mathrm{x}$ & $\mathrm{x}$ & & \\
\hline Ziziphus spina- & $\mathrm{x}$ & & $\mathrm{x}$ & $\mathrm{X}$ & $\mathrm{x}$ \\
\hline
\end{tabular}

\section{Analyse des indicateurs de pression}

L'analyse des différentes formes de pression montre que pour l'ensemble des indicateurs, les fréquences sont plus élevées au niveau des camps des réfugiés qu'au niveau des villages autochtones. Ce même résultat montre que les premières auréoles, notamment proches des villages et des camps de réfugiés sont les plus affectées. En effet, dans la première auréole, $25,3 \%$ des arbres sont totalement coupés contre $1,4 \%$ au niveau des villages autochtones. Au niveau de ceux-ci en effet, aucun arbre partiellement coupé n'a été observé (Tableau 3). L'analyse des fréquences de l'émondage au niveau des camps de réfugiés montre que 24,09\% des arbres sont émondés à des taux compris entre 25 et $75 \%$ de leur houppier dans la première auréole. Dans la même auréole, plus de 20,4\% des arbres sont émondés à plus de $75 \%$ de leur houppier, alors qu'au niveau des villages autochtones aucun arbre n'a été observé dans cette classe.

Tableau 3. Intensité de pression sur les espèces ligneuses

\begin{tabular}{|c|c|c|c|c|c|c|}
\hline \multirow{2}{*}{ Niveaux } & \multicolumn{2}{|c|}{ Intensité des coupes (\%) } & \multicolumn{3}{c|}{ Émondage (\%) } & \multirow{2}{*}{$\begin{array}{c}\text { Écorçage } \\
(\%)\end{array}$} \\
\cline { 2 - 6 } & Totalement & Partiellement & $<25$ & $25-75$ & $>75$ & \\
\hline \multicolumn{7}{|c|}{ Camps des réfugiés } \\
\hline Auréole 1 & 25,3 & 13,6 & 10,5 & 24,09 & 20,4 & 13,6 \\
\hline Auréole 2 & 11,4 & 9,1 & 8,5 & 18,4 & 16,09 & 2,3 \\
\hline Auréole 3 & 1,7 & 2,6 & 3,3 & 10,4 & 11,4 & 0 \\
\hline \multicolumn{7}{|c|}{ Villages autochtones } \\
\hline Auréole 1 & 1,4 & 0 & 2,9 & 2,5 & 0 & 0,4 \\
\hline Auréole 2 & 0,1 & 0 & 2,08 & 2,2 & 0 & 0,1 \\
\hline Auréole 3 & 0 & 0 & 1,5 & 0,8 & 0 & 0 \\
\hline
\end{tabular}

\section{Analyse du gradient de pression}

Les paramètres dendrométriques calculés et les variables mesurées en fonction des différentes auréoles ont été soumis à une Analyse en composantes principales (ACP). Les résultats de l'analyse montrent que le premier axe concentre 94,6\% des variances totales (Figure 4). L'observation du plan factoriel montre que cet axe constitue le gradient de pression. De la première auréole à la troisième, l'intensité de pression augmente. Dans la première auréole, les peuplements ligneux sont dominés par des individus âgés avec des valeurs élevées de la hauteur et du diamètre. Les facteurs de pression, notamment l'écorçage, l'émondage et la coupe de toutes intensités sont observés sur les arbres de la première auréole, proche des camps de réfugiés. 
On constate que la densité des adultes, la densité de la régénération, le taux de recouvrement et le taux de régénération sont plus élevés au niveau de la troisième auréole. La deuxième auréole présente des caractéristiques intermédiaires.

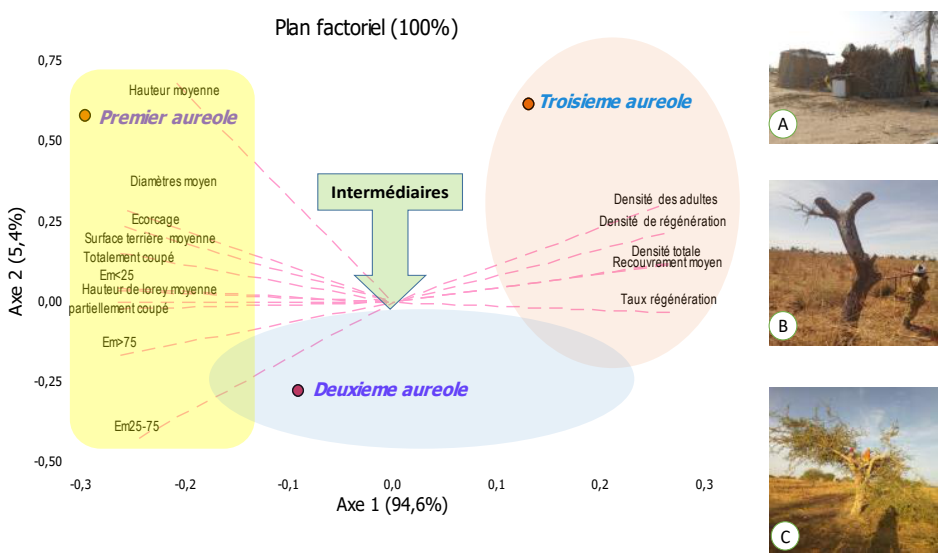

Figure 4. Répartition des paramètres dendrométriques suivant les auréoles. A : Maison des réfugiés ; B : Arbre d'Acacia tortilis coupé ; C : Arbre de Balanites aegyptiaca émondé.

\section{Distribution des espèces ligneuses}

L'analyse de la distribution des espèces ligneuses suivant les auréoles montre qu'au niveau global, Acacia tortilis est l'espèce la plus dense avec 12 tiges par ha, suivie de l'espèce Balanites aegyptiaca avec 8,3 tiges par ha. La densité totale de la zone est de 34,7 tiges par ha. L'analyse du tableau 4 montre qu'à l'échelle des auréoles, la densité totale augmente de la première à la troisième auréole. Elle est de 18 tiges par ha au niveau de la première, de 24 tiges par ha au niveau de la deuxième et de 62 tiges par ha au niveau de la troisième c'est-à-dire loin des camps. Concernant les espèces, il est noté que seule Acacia tortilis est présente dans toutes les auréoles. Par contre, Maerua crassifolia, Boscia senegalensis et Salvadora persica ont disparu de la première auréole. Hyphaene thebaica et Prosopis juliflora disparues de la première et de la deuxième auréole, sont observées au niveau de la troisième auréole, c'est-à-dire loin des camps et des villages (Tableau 4).

Tableau 4. Distribution des espèces ligneuses suivant les auréoles

\begin{tabular}{|l|c|c|c|c|c|c|c|c|}
\hline \multirow{2}{*}{ Espèces } & \multicolumn{2}{|c|}{$1^{\text {ère }}$ auréole } & \multicolumn{2}{c|}{$2^{\mathrm{e}}$ auréole } & \multicolumn{2}{c|}{$3^{\mathrm{e}}$ auréole } & \multicolumn{2}{c|}{ Globale } \\
\cline { 2 - 11 } & $\begin{array}{c}\text { Densité } \\
\text { (tiges/ha) }\end{array}$ & $\%$ & $\begin{array}{c}\text { Densité } \\
\text { (tiges/ha) }\end{array}$ & $\%$ & $\begin{array}{c}\text { Densité } \\
\text { (tiges/ha) }\end{array}$ & $\%$ & $\begin{array}{c}\text { Densité } \\
\text { (tiges/ha) }\end{array}$ & $\%$ \\
\hline Acacia tortilis & 6 & 25 & 12 & 50 & 18 & 29 & 12,0 & 38,9 \\
\hline Balanites aegyptiaca & 6 & 50 & - & - & 19 & 30,6 & 8,3 & 28,7 \\
\hline Maerua crassifolia & - & - & 5 & 20,8 & 13 & 21 & 6,0 & 13 \\
\hline Boscia senegalensis & - & - & 5 & 20,8 & 7 & 11,3 & 4,0 & 7,4 \\
\hline Leptadenia pyrotechnica & 4 & 16,7 & - & - & - & - & 1,3 & 3,7 \\
\hline
\end{tabular}




\begin{tabular}{|l|c|c|c|c|c|c|c|c|}
\hline Acacia senegal & 1 & 4,2 & 1 & 4,2 & - & - & 0,7 & 1,9 \\
\hline Salvadora persica & - & - & 1 & 4,2 & 1 & 1,6 & 0,7 & 1,9 \\
\hline Hyphaene thebaica & - & - & - & - & 2 & 3,2 & 0,7 & 1,9 \\
\hline Prosopis juliflora & - & - & - & - & 2 & 3,2 & 0,7 & 1,9 \\
\hline Ziziphus mauritiana & 1 & 4,2 & - & - & - & - & 0,3 & 0,9 \\
\hline Total & 18 & 100 & 24 & 100 & 62 & 100 & 34,7 & 100 \\
\hline
\end{tabular}

\section{Dynamique de l'occupation des sols de la commune de Chétimari}

La carte d'occupation du sol en 2002 et 2017 de la commune rurale de Chétimari montre que l'espace communal présente sept (7) unités physiographiques (Figure 5 et 6) composées de sol nu, sol sableux nu, steppe arbustive intacte, steppe arbustive dégradée, de la forêt galerie, du cordon ripicole et zone de culture. L'analyse de l'évolution de l'occupation des sols de la commune de Chétimari dans la période de l'étude montre une réduction des superficies de la steppe arborée. Celle-ci est passée de 59,3\% (79758,5 ha) en 2002 à 19,5\% (26227,5 ha) en 2017, soit une réduction de 40,28\% (54176,6 ha). De même, les cordons ripicoles et les galeries forestières ont connu une réduction de 3,25\% (4371,2 ha) de leur superficie. Par ailleurs, des augmentations des superficies sont aussi enregistrées dans d'autres strates tels que $17,32 \%$ (23295,4 ha) pour les steppes arborées dégradées, 9,73\% (13086,8 ha) pour les sols nus et $0,88 \%$ ( 1183,6 ha) pour les zones de culture.

Dynamique paysagère de la Commune de Chétimari entre 2002 et 2017

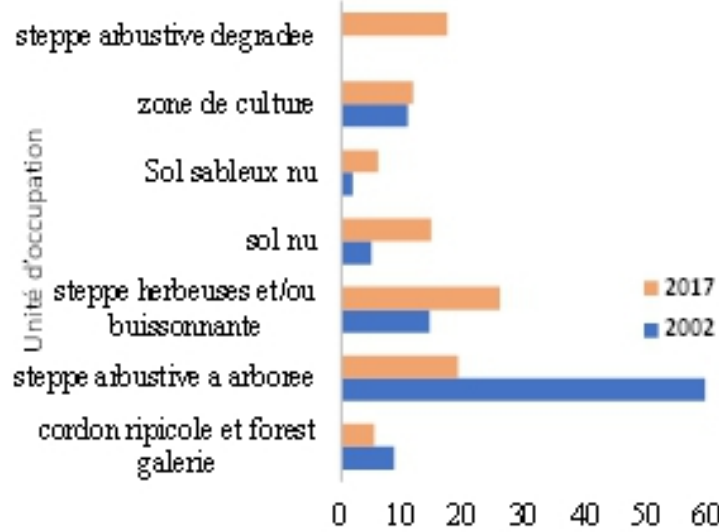

Pouroentage de la tache $(\%)$

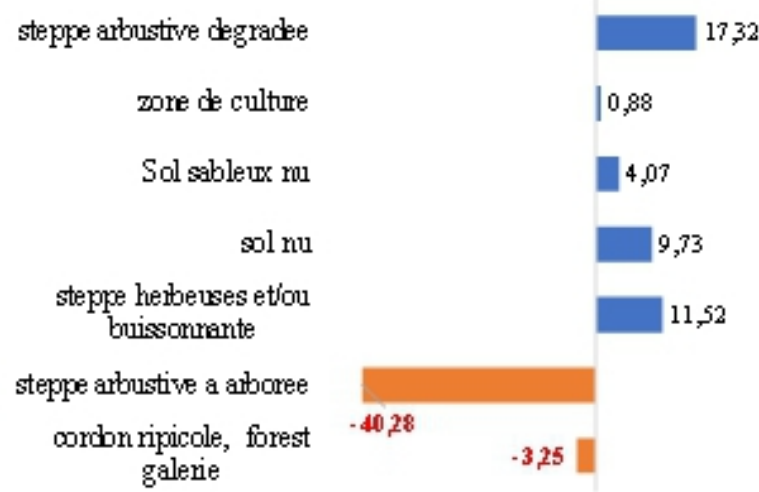

$-45,00 \quad-25,00 \quad-5,00 \quad 15,00$

Figure 5. Dynamique de l'occupation des sols de la commune de Chétimari 

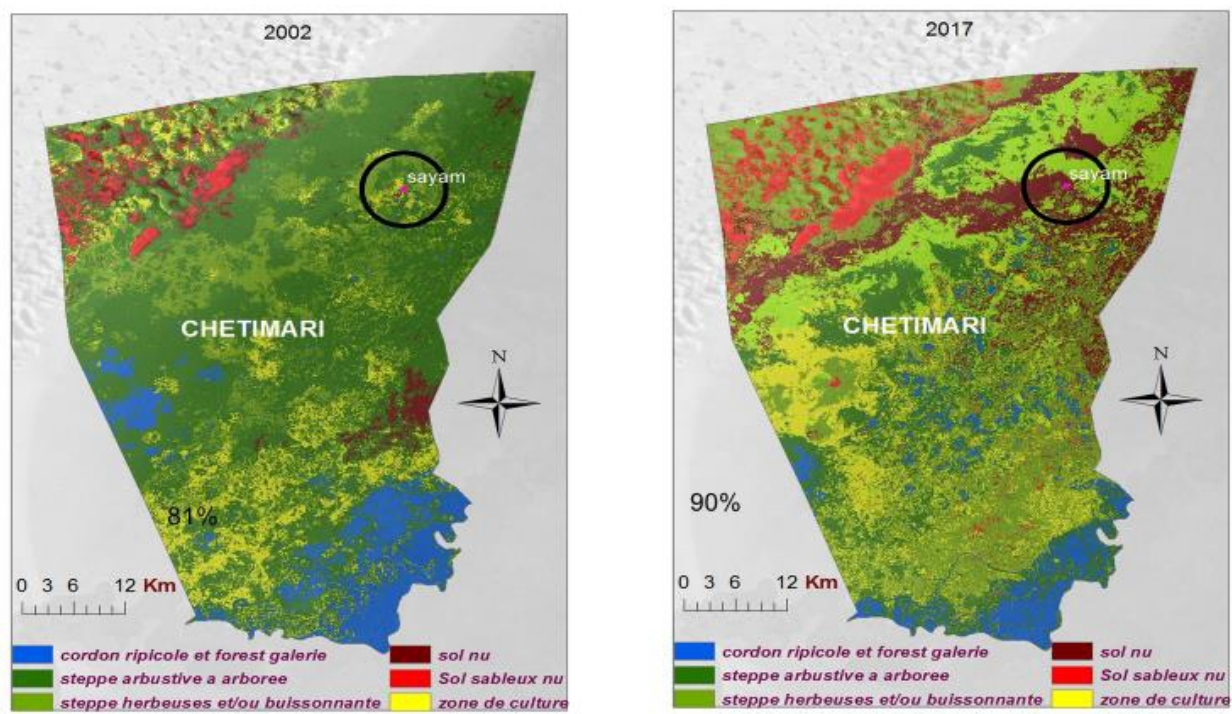

Figure 6. Cartes d'occupation des sols de la commune de Chétimari en 2002 et 2017

\section{Dynamique de l'occupation des sols de la commune de Gueskérou}

La carte d'occupation du sol en 2013 et en 2017 de la Commune rurale de Gueskérou montre que l'espace communal présente neuf (9) unités physiographiques (figure 7 et 8) composé de zone de culture pluviale, de sol nu, de steppe arbustive, de steppe arbustive dégradée, de zones d'habitation, de zones de culture maraichère de la forêt galerie, du cordon ripicole et du plan d'eau. L'analyse de la dynamique de l'occupation des sols de la commune de Geskérou montre une diminution de la steppe arbustive. Ces superficies évaluées à 42,8\% (84358,8 ha) en 2013 ont passé à 36,4\% (71744,4 ha) en 2017 soit une réduction de 6,49\% (12791,7ha). La steppe arbustive dégradée qui était de 15,6\% (30747,6 ha) en 2013 a passé à 26,2\% (51640,2 ha) en 2017 soit une augmentation de 10,6\%. (20892,6 ha) Les steppes arbustives et les zones de cultures ont connu une réduction entre 2013 et 2017. Les sols nus qui étaient de 4,1\% (8081,1 ha) en 2013 ont passé à 8,09\% (15945,3 ha) en 2017 soit une augmentation de 4,08\% (8041,6 ha). 
Dynamique paysagère de la Commune de Gueskérou entre 2013 et 2017
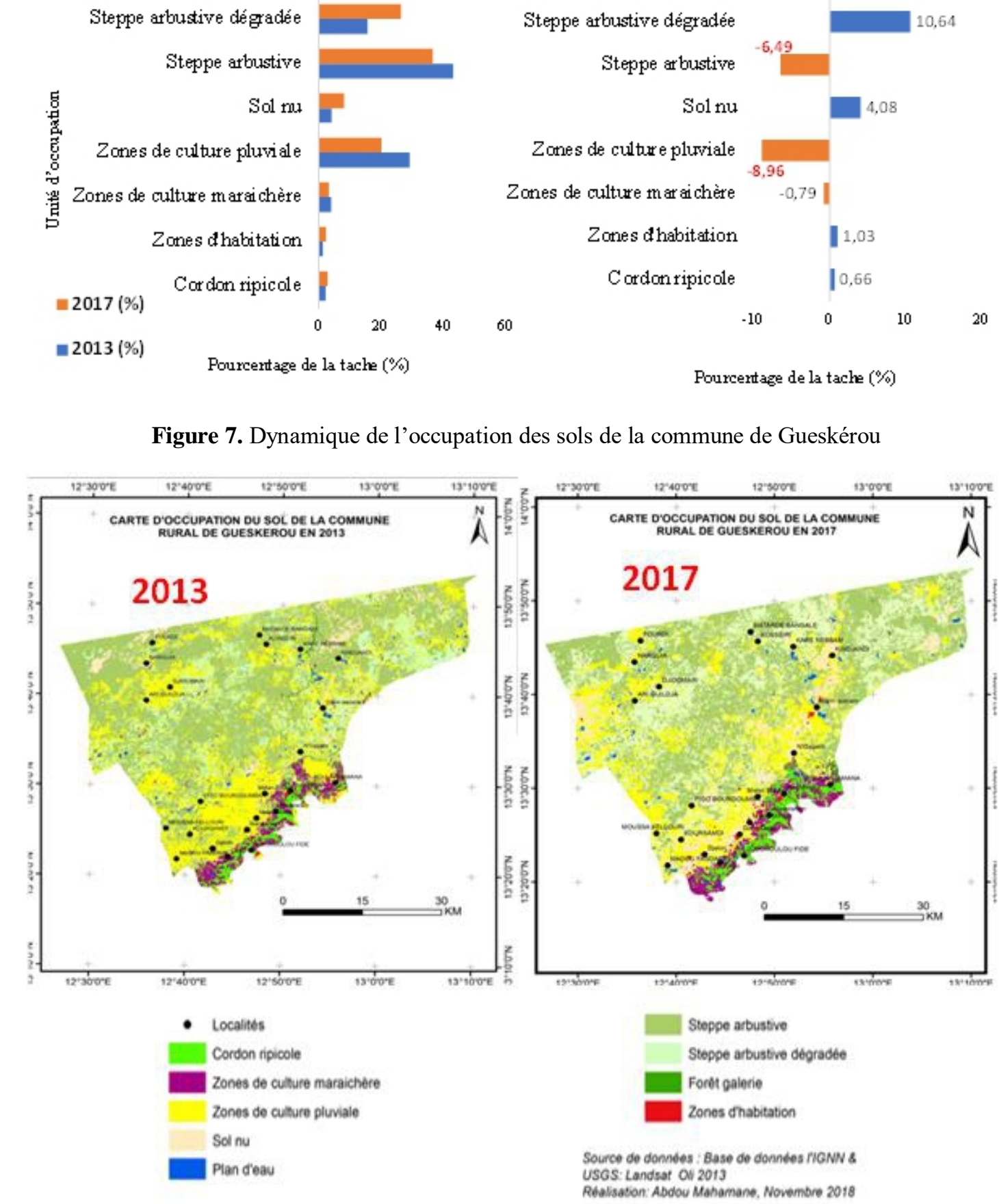

Pourcentage de la tache (\%)

Figure 7. Dynamique de l'occupation des sols de la commune de Gueskérou

Figure 8. Carte de la dynamique de l'occupation des sols en 2013 et 2017 de la commune de Gueskérou 


\section{Discussion}

\section{Diversité et importance sociale des ressources ligneuses}

Les espèces ligneuses jouent des rôles très importants dans la vie des populations de la zone d'étude. Elles assurent de multiples et divers usages pour la survie des populations particulièrement en zones rurales (Laminou et al., 2017 ; Rabiou et al., 2017). Les auteurs (Hiernaux et al. 2009) ont évoqué un certain nombre d'usages parmi lesquels on peut citer l'alimentation humaine, bois énergie, le bois de service, les plantes médicinales, le fourrage vert et des rôles écologiques importants comme l'atténuation des changements climatiques et la fertilisation des sols. De plus, Hamidou et al. (2013) ont montré que la production du bois est l'un des rôles importants attribué aux végétaux ligneux qui motive les paysans à entretenir les arbres dans leurs champs (Hamidou et al., 2013).

Suite à la crise de Boko Haram, les sites des réfugiés de la commune de Gueskérou ayant fait l'objet de la présente étude ont accueilli 42368 personnes déplacées soit plus de 50\% de la population autochtone (UNHCR, 2017). En s'installant, ces déplacés ont eu besoin de fourches, de traverses et de piliers pour la construction de leurs abris. Faute d'appui des œuvres humanitaires dans ce domaine, les réfugiés, sans aucune connaissance des méthodes de sélection, ont fait recours à la coupe anarchique du bois vert issu du maigre potentiel ligneux déjà fragilisé par les facteurs climatiques (Diallo, 2015). Dans le cadre de la présente étude huit (8) espèces ont été recensées dans la commune de Gueskérou comme étant les espèces les plus exploitées. Il s'agit de Balanites aegyptiaca, Acacia senegal, Acacia tortilis, Hyphaene thebaica, Leptadenia pyrotechnica, Prosopis juliflora et Ziziphus mauritiana. L'exploitation et le prélèvement portent essentiellement sur les tiges mais aussi les racines pour les arbres. Les parties prélevées sont utilisées dans la confection des outils aratoires notamment les manches pour la hilaires, la houe pour les travaux champêtres. Ces produits font d'ailleurs l'objet de vente sur les camps des réfugiés et constituent une source complémentaire de revenus. Plusieurs auteurs au Niger ou dans la sous-région ont montré l'importance de ces plantes dans la vie socioéconomique des population (Douma, 2008 ; Rabiou et al., 2017 ; Halimatou, 2010 ; Mahamane et al., 2009 ; Saidou et al., 2015).

L'amélioration de la fertilité des sols constitue un apport des arbres dans les champs et cela guide les cultivateurs dans le choix des espèces à gérer. Les cultivateurs reconnaissent que certaines espèces ligneuses améliorent la fertilité des sols (Sofiani, 2016). C'est ainsi que dans les deux sites, les espèces maintenues dans les champs pour assurer ce rôle de fertilisation sont : Faidherbia albida et Balanites aegyptiaca. Les populations ont recours aux plantes médicinales en raison de la très faible couverture sanitaire en zones rurales au Niger (Idi-Ounani, 2013), particulièrement dans la vallée du Lac 
Tchad (AFD, 2018). Elles sont exploitées par écorçage, arrachage des feuilles et par déracinement. L'exploitation des organes touche l'écrasante majorité des espèces. Parmi les espèces les plus exploitées on peut citer Balanites aegyptiaca, Acacia senegal, Ziziphus mauritiana, Leptadenia pyrotechnica, Faidherbia albida et Acacia nilotica (Douma, 2008).

Les arbres occupent une place importante dans la stratégie de l'amélioration de la sécurité alimentaire. Le recours aux produits forestiers non ligneux, notamment les feuilles et les fruits, s'observe le plus souvent pendant les périodes de soudure alimentaire correspondant à l'approche de la saison des pluies où l'exploitation devient plus intense (Douma, 2008 ; Rabiou et al., 2014). Dans les deux communes ayant fait l'objet de la présente étude et dans toute la vallée du Lac Tchad au Niger, les espèces utilisées dans l'alimentation humaine sont Ziziphus mauritiana, Balanites aegyptiaca, Maerua crassifolia, Boscia senegalensis, Acacia senegal. Les espèces ligneuses les plus utilisées comme fourrage sont Acacia tortilis, Faidherbia albida, Ziziphus mauritiana, Balanites aegyptiaca et Acacia senegal. L'utilisation de ces espèces comme plantes fourragères a été signalée par plusieurs auteurs. On peut citer les travaux de Ndong et al. (2015) et Sarr et al. (2013) dans le sahel du Sénégal et les travaux de Silue et al. (2014) au nord de la Côte d'ivoire.

Selon plus de 98\% de personnes (autochtones) enquêtées, l'arrivée massive des personnes déplacées ou refugiées a eu un impact très négatif sur l'état des peuplements ligneux notamment les parcs agro-forestiers, les parcours pastoraux et les champs de cultures. L'installation des camps des réfugiés de Boudouri et de Maina Kaderi a privé certaines personnes de leurs terres de cultures (UNHCR, 2017). Pourtant, les principales activités sources de revenus des ménages dans la zone d'étude sont l'agriculture, l'élevage, le petit commerce et autres. En effet, l'installation a privé certaines personnes (autochtones) de leurs champs. En plus, la totalité des champs cultivées par les refugiées ou déplacés est soit empruntée soit des jachères ou des parcours pastoraux. Ceci a augmenté la pression sur les champs de cultures entrainant la régression des superficies cultivées, le raccourcissement des périodes de jachère et diminution des espaces pastoraux exacerbant ainsi les conflits entre éleveurs et agriculteurs. Cette situation a aussi contraint les éleveurs à exercer plus de pression sur les espèces ligneuses à travers l'émondage sévère pour combler le déficit fourrager. S'agissant de parcs agroforestiers, les arbres sont victimes des coupes abusives malgré la mise en défens et le suivi régulier des agents de service de l'environnement (MESU/DD, 2014). Les arbres sont coupés et écorcés nuitamment afin de contourner la vigilance des propriétaires des champs et au mépris des lois régissant l'exploitation des ressources forestières. Quant à la densité des ligneux, il a été affirmé à l'unanimité (autochtones et refugiés) que la strate ligneuse de la zone était très dense avant 
l'arrivée des réfugiés. Actuellement, suite à la pression on constate l'apparition des sols nus exposés ainsi à l'érosion. Les raisons principales avancées par les réfugiés pour justifier cette forte exploitation est la précarité de leur situation économique qui les a contraint à abattre les arbres principalement pour la construction d'abris et bois énergie qui est la principale source d'énergie de foyers même chez les autochtones. Les espèces les plus abattues sont les Balanites aegyptiaca, Acacia tortilis, Boscia senegalensis et Acacia senegal du fait de leur abondance (soient 266,4 pour 300 en termes de IVI pour les 4 espèces). Ces dernières espèces sont menacées face à la pression climatique et anthropique en cours qui ne cesse de s'amplifier ainsi que l'ont observé d'autres auteurs (MHA, 2012 et 2017). Le besoin continu en terres cultivables et le déboisement continuel ont sensiblement changé le paysage au cours des dernières années, avec une forte régression en nombre d'espèces ligneuses, même si l'impact précis de l'arrivée des réfugiés est encore difficilement estimable compte tenu notamment de l'insuffisance de données existantes avant leur arrivée (Morou et al., 2016 ; OXFAM, 2016).

\section{Impact de l'exploitation sur les ressources ligneuses}

Globalement les paramètres dendrométriques tels que les densités, le recouvrement, la surface terrière, le diamètre moyen, la hauteur moyenne, et la hauteur de Lorey sont relativement très faibles par rapport aux résultats rapportés par plusieurs études en zone sahélienne (Mamadou, 2016 ; Hamidou et al., 2013 ; Saidou et al., 2015 ; Idi-Ounani, 2013). Les caractéristiques dendrométriques varient significativement d'un site à un autre et d'une auréole à une autre. Cette différence pourrait être due au nombre important des déplacés qu'accueillent les camps de réfugiés des communes de Chétimari et Gueskérou. Il est évident qu'une forte densité de population associée à la fragilité écologique des écosystèmes où s'installent les réfugiés est un des facteurs primordiaux de risques de dégradation de l'environnement (Richard, 1998).

L'analyse des caractéristiques dendrométriques révèle des différences significatives suivant les auréoles pour la plupart des paramètres (les densités, taux de régénération et le recouvrement). Ces paramètres sont très faibles dans la première auréole (18 arbres à l'hectare), moyens dans la deuxième auréole (24 arbres à l'hectare) et très élevés dans la troisième auréole (62 arbres à l'hectares). Ce qui traduit la forte pression exercée sur les ressources ligneuses dans les environnements immédiats des sites. Il ressort de l'observation faite sur les individus que la totalité des arbres dans la première auréole du site de Boudouri et celle du site de Maina Kaderi sont victimes au moins en partie, d'une des pressions (émondage, coupe, écorçage). Parmi ces pressions, les plus fréquentes sont l'émondage à plus de $50 \%$ de houppier suivies, des coupes (totales et partielles) et enfin l'écorçage. Cette dernière pression est très 
insignifiante $(5 \%)$ dans la première auréole du village de Malam Gamari. Cet écart important de l'intensité des pressions entre les camps de réfugiés et les villages autochtones, et les différentes auréoles pourrait s'expliquer par l'abattage incontrôlé d'arbres pour le bois de chauffe ou pour des fins de construction d'abris et de vente. Cet état de fait a été décrié dans plusieurs rapports internationaux (UNHCR, 2013 et 2017 ; Richard, 1998) au Tchad, au Rwanda, au Kenya, au Congo et au Burundi. Selon le Programme alimentaire mondial (PAM) cité par Richard (1998), l'impact potentiel des réfugiés sur les environnements locaux est énorme, tout particulièrement le déboisement autour des installations de réfugiés au Pakistan et au Malawi. Ces résultats confirment le récent rapport de UNHCR (2017) dans la région de Diffa sur l'audit environnemental et social qui fait état de coupe abusive de bois par les déplacés (refugies, déplacés, retournés).

\section{Impact de l'exploitation des ressources ligneuses sur la dynamique paysagère}

L'évaluation de la dynamique de l'occupation du sol de la commune rurale de Gueskérou met en exergue des changements au niveau de chacune des unités d'occupation retenues. Dans cette période de 4 ans (2013-2017) correspondant aux périodes d'occupation des espaces par les réfugiés fuyant les exactions de Boko Haram dans le lit du Lac Tchad, on constate des tendances évolutives (gain au détriment d'un autre type d'occupation du sol) et régressives (perte de superficie au profit d'un autre type d'occupation du sol). Les principales tendances observées au niveau de cette Commune sont : une diminution des superficies de la steppe arbustive, de la forêt galerie, des zones des cultures pluviales et des cultures maraichères, une augmentation de la superficie des steppes arbustives dégradées, des sols nus, des zones d'habitation, de cordon ripicole. L'un des faits marquants des changements intervenus dans l'intervalle de quatre (4) ans, dans la commune de Gueskérou est la dégradation de la végétation naturelle. Cette transformation à l'échelle de 4 ans est la résultante des actions humaines telles que les activités de déboisement, du surpâturage consécutives à un accroissement fulgurant de la population de la commune qui a enregistré un flux massif des personnes déplacées et refugiées. En effet, avec la crise de Boko Haram, l'effectif de la population est passé de 40923 à 123995 habitants de 2013 à 2017. Par ailleurs le déplacement de plusieurs villages riverains de la rivière Komadougou Yobé a entrainé l'abandon des champs qui a une répercussion sur la diminution des zones des cultures pluviales et irriguées. De plus, l'installation des personnes déplacées a contribué à l'agrandissement des villages d'accueil. Il en résulte une augmentation des aires d'habitation DREC/MR, 2017). 


\section{Conclusion Et Suggestions}

L'étude a permis de mettre en évidence la forte anthropisation sur les maigres ressources ligneuses de la région de Diffa au Niger imputable à l'installation des personnes déplacées et refugiées victimes de la crise Boko Haram. Les principaux facteurs de pression sont essentiellement la collecte quotidienne de bois énergie, la production du charbon, la coupe du bois d'œuvres et de services, la cueillette des produits et organes des ligneux et l'exploitation du fourrage. Les effets de cette forte anthropisation sur les ressources ligneuses déjà affectées par la désertification se traduisent par la formation des auréoles de déforestation dont le rayon s'accroît chaque année, la perte de la diversité ligneuse, la conversion de l'occupation des sols, la formation des sols nus favorisant ainsi l'érosion éolienne et hydrique, une régression des superficies de la steppe arbustive et de la forêt galerie, une extension des steppes arbustives dégradées et la formation des dunes de sable. L'impact de l'installation des camps des réfugiés sur l'environnement constitue une préoccupation dans la région de Diffa, une région déjà affectée par la désertification et le changement climatique.

$\mathrm{Au}$ terme de cette étude nous formulons les suggestions suivantes en vue de protéger les écosystèmes de la région de Diffa. Il s'agit de : promouvoir des abris sans bois; accentuer la distribution des bouteilles à gaz et sensibilisation sur leur mode d'emploi; créer des points de recharge de bouteilles à gaz dans tous les sites ; organiser des activités de restauration des terres et plantation des arbres (reboisement) ; créer des activités génératrices de revenus à l'endroit des personnes déplacées et autochtones ; organiser des campagnes de sensibilisation sur la protection de l'environnement ; interdire les coupes abusives de bois.

\section{References:}

1. Adamou L. O. et Adamou M. S., 2018. Impact de l'installation de camps de réfugiés sur les ressources ligneuses cas de la commune de Chétimari. Mémoire de licence, Université de Diffa au Niger, 52p.

2. AFD (Agence Française de Développement), 2018. Crise et développement : la Région du Lac Tchad à l'épreuve de Boko Haram, 294 p.

3. Congalton R.G., 1991. A review of assessing the accuracy of classification of remotely sensed data. Remote Sens. Environ. 37:3546.

4. Diallo A., 2015. Impacts environnementaux des refugies autour des zones conflictuelles en Afrique de l'Ouest, Mémoire de Master de recherche, Option : gestion des ressources naturelles. Université Ouaga I, Burkina Faso, 85 p. 
5. Douma S., 2008. Écologie de quatre espèces ligneuses de la Réserve Totale de faune de Tamou: indicateurs de gestion durable des ressources forestières, Mémoire de DEA, UAM, 89P.

6. DREC/MR (Direction Régionale de l'État Civil, des Migrations et Réfugiés), 2017. Rapport de recensement des réfugiés, déplacés et retournés dans la Région de Diffa, 25p.

7. Enaruvbe G.O.; Atedhor G.O., 2015. Spatial Analysis of Agricultural Landuse Change in Asaba, Southern Nigeria. Ife Journal of Science. 17(1):65-74.

8. FAO., 2012. Situation des forêts du monde. Organisation des Nations Unies pour l'Alimentation et l'Agriculture Rome, $66 \mathrm{p}$.

9. FAO, 1994. Introduction à la gestion conservatoire de l'eau, de la biomasse et de la fertilité des sols (CGES). Bulletin Pédologique de la FAO n ${ }^{\circ} 70,420 p$;

10. Forkuo E.K.; Frimpong A., 2012. Analysis of Forest Cover Change Detection. International Journal of Remote Sensing Applications. 2(4):82-92.

11. Rabiou H., Bationo B. A.., Kossi A., Adzo Dzifa K.,Ali M., et Kouami K., 2017. Perception paysanne et importance socioculturelle et ethnobotanique de Pterocarpus erinaceus au Burkina Faso et au Niger. Afrique Science 13(5) (2017) $43-60$.

12. Halimatou B., 2010. Caractérisation biophysique des ressources ligneuses dans les zones dégradées et reverdies au Sahel : cas du département de Mayahi mémoire Diplôme d'Etudes Approfondies en Biologie appliquée, Université Abdou Moumouni de Niamey, Niger, 14-18p.

13. Hamidou A., Boubé M., Rabiou H., Mahamane A., 2013. Caractéristiques floristiques, diversité et structure de la végétation ligneuse dans le Centre-Sud du Niger : cas du complexe des forêts classées de Dan kada Dodo-Dan Gado. Int. J. Biol. Chem. Sci., 3(7) : 1048-1068.

14. Idi-Ounani H., 2013. Évaluation Des Ressources Forestières Et Leur Mode De Gestion Dans Le Terroir De Dan Saga (Aguié, Niger). Mémoire de master, Université d'Abomey Calavi, 89p.

15. Hiernaux P., Diarra L., Trichon V., Mougin E., Soumaguel N., Baup F., 2009, Woody plant population dynamics in response to climate changes from 1984 to 2006 in Sahel (Gourma, Mali). Journal of Hydrology, 375 (1-2), 103-113.

16. INS, 2012. $4^{\mathrm{e}}$ recensement général de la population du Niger, 186p.

17. Kiari A. K., Ousmane Laminou M., Iro Dan G., Saley K., Rabiou H., Roger P., 2017. Diversité floristique et structure de la végétation dans 
la zone dunaire du sud-est du Niger : Cas de Mainé soroa. Journal of Applied Biosciences, 120: 12053-12066.

18. Laminou M. O., Boubé M., Saley K., Oumarou Bachir G., Ali M. 2017. Usages Socioeconomiques Des Espèces Ligneuses Au Sahel: Cas De Guidan Roumdji Au Niger, European Scientific Journal, 26(13) $1857-7881$.

19. Maazou R., Rabiou H., Youssoufa I., Lawali., Sani IDI S., Mahamane A., 2017. Influence de l'occupation des terres sur la dynamique des communautés végétales en zone Sahélienne : cas de la commune rurale de Dantchandou (Niger), Int. J. Biol. Chem. Sci., 11(1): 79-92.

20. Mahamane A., Saadou M., 2009. Méthode d'étude et d'analyse de la flore et végétation tropicale 25-32p.

21. Mahamane A., Saadou M., Mohamed B. D., Karim S., Bakasso Y., Abdoulaye D., Morou B., Inoussa M. M., Idrissa S., Arzika T., 2009. Biodiversité végétale au Niger : État des connaissances actuelles. Annale de l'Université de Lomé, Séries Sciences, Tome XVIII : 81-93.

22. Mahamane M., 2015. Assassing soil erosion risk in the Tillabery landscape Niger. African Journal of Environmental Scinece and Technology, Vol. 9(3) : 176-191.

23. Mahamane M., Hochschild V., Schultz A., Kuma J., 2015. Monitoring Desertification in the Tillabery Landscape (Sahel Region) using Change Detection Methods and Landscape Metrics, International Journal of Applied Research, Vol. 1(10) : 315-321 :

24. Mamadou M., 2016. Étude des incidences de déplacement des populations fuyant les exactions de Boko-Haram sur les composantes environnementales et agricoles dans la Région de Diffa : Cas des sites de Boudouri et Maina-kaderi dans la commune rurale de Chétimari mémoire de master, Université de Diffa, $69 \mathrm{p}$.

25. Manandhar R.; Odeh, I. O. A.; Ancev T., 2009. Improving the Accuracy of Land Use and Land Cover Classification of Landsat Data Using Post-Classification Enhancement. Remote Sens.1:330-344; DOI:10.3390/rs1030330.

26. MESU/DD (Ministère de l'Environnement, de la Salubrité Urbaine et du Développement Durable), 2014. Cadre Stratégique de la Gestion Durable des Terres au Niger (CS-GDT) et son plan d'investissement 2015-2029, 100p.

27. MHA (Ministère de l'Hydraulique et de l'Assainissement), 2012. Plan Forestier National (PFN)-Niger 2012-2021, 98p.

28. MHA (Ministère de l'Hydraulique et de l'Assainissement), 2017. Plan Local Eau et Assainissement (PLEA) de la Commune de Gueskerou, $66 \mathrm{p}$. 
29. Morou B, Amadou OA, Sitou 1, Ali., 2016. Ressources forestières ligneuses : diversité et usages dans le terroir villageois de Dan Saga. $13 p$.

30. Ndong A. T., Ndiaye O., Ngom Faye M., Galop D. et Guissé A., 2015. Espèces ligneuses du Ferlo-Nord, Sénégal : état actuel et usage. Les Cahiers d'Outre-Mer, 271 : 285-462.

31. OXFAM., 2016, EMMA (Analyse et cartographie des marchés en état d'urgence) modifiée et analyse de la protection. Les systèmes de marché des revenus du poisson fumé et du poivron rouge séché, Région de Diffa, Niger oriental, 46 p.

32. PDC (Plan de Développement Communal), 2010. Rapport de Commune Rurale de Gueskérou, 101p.

33. PDC (Programme d'Actions Communautaires), 2012. Commune Rurale De Chetimari. 48p.

34. Rabiou H., Inoussa Maman M., Bakasso Y., Diouf A., Mamoudou Moussa B., Mahamane A., Idi Saidou S., Saadou M., Anne Mette L., 2014. Structure de la population de Boscia senegalensis (Pers) Lam. Ex Poir suivant la toposéquense dans la commune de Simiri (Niger) Journal of Animal \&Plant Sciences, 2014. 3(23) : 3657-3669.

35. Rabiou H., Maazou R., Soumana I., Moussa Mamoudou B., IssaharouMatchi I., Mahamane A., 2017. Succession des communautés végétales des jachères protégées en zone sahélienne : cas de Banizoumbou (Niger) Journal of Applied Biosciences 111: 1094410956.

36. Richard B., 1998. L'impact des réfugiés sur l'environnement écologique des pays d'accueil (Afrique subsaharienne) Autrepart, $\mathrm{N}^{\circ}$ 7, p. 23-42.

37. Saadou M., 1990, La végétation des milieux drainés nigériens à l'Est du fleuve Niger. Thèse d'Etat, Université de Niamey, 395p.

38. Saidou O., Riccardo F., Hamani M., Alhassan Y : 2015. Diversité, structure et régénération de la végétation ligneuse de la Station Sahélienne Expérimentale de Toukounous, Niger Int. J. Biol. Chem. Sci. 9(2): 910-926

39. Samaali H., 2011. Etude de l'évolution de l'occupation et de l'utilisation du sol dans le delta de Mejerda par télédétection et systèmes d'informations géographiques. Thèse du Doctorat en Géographie, Université de Tunis, Tunis(Tunisie), 390p .

40. Sarr O., Diatta S., Gueye M., Ndiaye P.M., Guisse A., Akpo L.E., 2013. Importance des ligneux fourragers dans un système agropastoral au Sénégal (Afrique de l'ouest). Revue Méd. Vét., 1(164) : 2-8.

41. Silue N., Fofana J. I., Silue S., Diarrassouba N., Kouassi A. F. Et Kouakou K., 2014. Identification des especes ligneuses utilisees dans 
l'alimentation des Bovins dans la Region du Poro (nord de la Côte D'ivoire). Agronomie Africaine, 26 (3) : 217 - 229.

42. Sinsin B., 1993. Phytosociologie, écologie, valeur pastorale, production et capacité de charge des pâturages naturels du périmètre Nikki- Kalalé au Nord-Bénin. Thèse de Doctorat en Sciences Agronomiques. Université Libre de Bruxelles, Belgique, 390 pp.

43. Sofiani S. D., 2016. Caractérisation fourragère du centre secondaire de multiplication de bétail de Fako (Nord-Dakoro/Niger) : capacité de charge, élaboration d'un plan d'aménagement et évaluation de son impact économique. Mémoire de Master, Université de Maradi, 45p.

44. UICN/UNHCR, 2005. Gestion forestière en contexte d'accueil de réfugiés. Rapport d'activités, $55 \mathrm{p}$.

45. UNEP/FAO. 1994. Report of the UNEP/FAO Expert Meeting on Harmonizing Land Cover and Land Use Classifications. Geneva, 2325 November 1993. GEMS Report Series, No. 25.

46. Suleiman Y.M.; Saidu S.; Abdulrazaq S.A.; Hassan A.B. Abubakar, A.N., 2014. The Dynamics of Land Use Land Cover Change: Using Geospatial Techniques to Promote Sustainable Urban Development in Ilorin Metropolis, Nigeria. Asian Review of Environmental and Earth Sciences. 1(1), 8-15.

47. UNHCR, 2013. Évaluation environnementale stratégique de l'installation des refugies maliens dans les provinces du seno, soum et oudalan (BURKINA FASO), 91p.

48. UNHCR, REACH, 2017. Site de Boudouri, Commune de Chetimari, région de Diffa, Niger, rapport hebdomadaire, 2p.

49. Youn L., 1985. A General Assessment of the Environmental Impact of Refugees in Somalia, with Attention to the Refugee Agricultural Programme, Disasters, $9: 122-133$. 